\title{
Gefitinib bei aktivierenden EGFR-Mutationen
}

Die Bedeutung von Biomarkern steigt auch beim Bronchialkarzinom, wie Michael Thomas, Heidelberg, betonte. So wird derzeit diskutiert, wann bei Patienten mit nichtkleinzelligem Bronchialkarzinom (NSCLC) eine Analyse des EGFR (Epidermal Growth Factor Receptor)-Mutationsstatus erforderlich ist.

Das ist vor allem eine Folge der Studie IPASS (Iressa Pan-Asia Study), so Thomas. In dieser Phase-III-Studie wurden rund 1.200 Patienten aus Asien mit NSCLC in der First-Line-Therapie entweder mit Carboplatin/Paclitaxel oder mit dem EGFRTyrosinkinase-Inhibitor Gefitinib therapiert (Mok TS et al., 2009, N Engl J Med 361: 947-957). Eingeschlossen wurden $\mathrm{Pa}$ tienten mit fortgeschrittenem Adenokarzinom ohne oder nur mit einer minimalen Raucheranamnese. Das Resultat war über die gesamte Studienpopulation hinweg ein signifikanter Vorteil beim progressionsfreien Überleben zugunsten der Therapie mit Gefitinib (Hazard-Ratio 0,74 [HR]; 95-\%-Konfidenzintervall [95-\%-KI] 0,65$0,85 ; p<0,001)$. Dieser Vorteil konnte auf das Therapieergebnis bei den Patienten mit aktivierende EGFR-Mutationen - rund $60 \%$ von 437 Patienten mit Tumorproben für die Biomarkeranalyse in dieser Studie zurückgeführt werden (HR 0,48; 95-\%-KI $0,36-0,64 ; p<0,001)$. In dieser Subgruppe lag das progressionsfreie Überleben bei der Therapie mit Gefitinib im Median bei 9,5 Monaten. Es betrug median 6,3 Monate, wenn Patienten mit aktivierenden EGFR-Mutationen Carboplatin/Paclitaxel erhielten.

Thomas betonte, dass der Anteil von Patienten mit aktivierenden EGFR-Mutationen in einem nichtselektierten Patientenkollektiv deutlich geringer ist. Ersten Zahlen zufolge liegt er bei $10 \%$ bis $15 \%$.

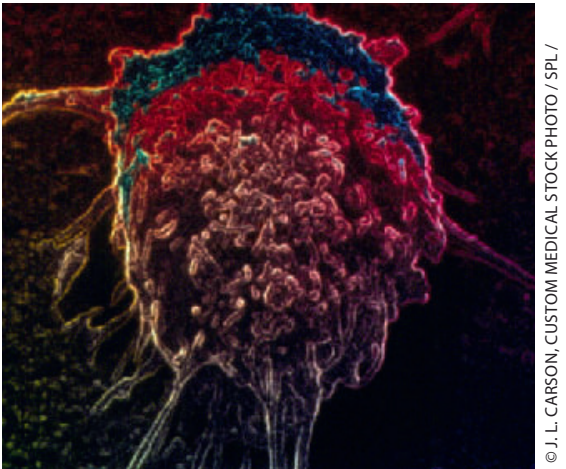

Adenokarzinomzelle, Bronchialkarzinom gefärbte rasterelektronenmikroskopische Aufnahme

Deutlich höher ist dieser Anteil bei Patienten mit Adenokarzinom und bei $\mathrm{Pa}$ tienten, die nicht oder selten rauchen. Denkbar wäre demnach, Patienten, bei denen eine EGFR-Mutationsanalyse gemacht wird, anhand dieser beiden klinischen Kriterien vorzuselektieren. $P G$

\section{Multiples Myelom}

\section{Pomalidomid bei Patienten mit rezidivierter oder refraktärer Erkrankung}

Beim multiplen Myelom könnte die Einführung einer neuen Substanz bevorstehen: Nach Thalidomid, Lenalidomid und Bortezomib dürfte bald Pomalidomid das therapeutische Armentarium ergänzen, so Lothar Kanz, Tübingen.

Zur Therapie mit dem Immunomodulatory Drug (IMiD) Pomalidomid gibt es gute Daten aus einer Ende 2009 publizierten Phase-II-Studie (Lacy MQ et al., 2009, J Clin Oncol 27: 5008-5014). In dieser Studie erhielten 60 Patienten mit rezidiviertem oder refraktärem multiplem Myelom täglich $2 \mathrm{mg}$ Pomalidomid in 28-Tages-Zyklen plus 40mg Dexamethason an den Tagen 1, 8, 15 und 22 in jedem Zyklus. Im Median wurden sieben Zyklen durchlaufen. Bei Progression oder inakzeptabler Toxizität wurde die Therapie abgebrochen. Insgesamt sprachen 63\% der Patienten auf die Therapie an, so Kanz. Besonders bemerkenswert dabei: Patienten, die zuvor refraktär auf eine Therapie mit Lenalidomid, Thalidomid oder Bortezomib waren, zeigten zu 40\%, 37\% und
60\% ein Ansprechen auf Pomalidomid (Tab.). „Damit dürften wir für diese Patienten bald eine neue Therapieoption in den Händen halten", sagte Kanz. Bisherigen Daten zufolge könne Pomalidomid beim multiplen Myelom als das effektivste immunmodulatorische Agens gelten. PG

Ansprechrate: Patienten mit refraktärer Erkrankung

Ansprechrate

Patienten mit refraktärer Erkrankung $(n=60) \quad 63 \%$

Bortezomib-refraktär $(n=10) \quad 60 \%$

Lenalidomid-refraktär $(n=20) \quad 40 \%$

Thalidomid-refraktär $(n=16) \quad 37,5 \%$

Bortezomib- und Lenalidomid-refraktär $(n=5) \quad 60 \%$

nach Lacy MQ et al., 2009, J Clin Oncol 27: 5008-5014 